\title{
Effects of Dietary Alkylglycerols in Lactating Rats on Immune Responses in Pups
}

\author{
SUK Y. OH AND LALITA S. JADHAV \\ Department of Dietetics and Nutrition, University of Kansas Medical Center, \\ Kansas City, Kansas 66103
}

\begin{abstract}
Alkylglycerols (AG) are glyceryl ether lipids and are present in human and cow's milk and the hematopoictic organs such as bone marrow, spleen, and liver. The biologic effects of AG include stimulation of blood leukocyte and thrombocyte production and activation of macrophage and anti-tumor activity. The present study was conducted to determine the effects of dietary AG in lactating rats on AG levels in milk and development of certain immune responses in the pups. Lactating rats were fed diets supplemented with various levels of AG. Milk samples were expressed from the dams and blood was collected from the pups on postpartum d 8,16, and 24. Concentrations of AG in milk from the dams fed $A G$ were significantly greater than those of the controls $(p<0.05)$. Peripheral blood granulocytes were significantly elevated in pups from the
\end{abstract}

ABSTRACT

dams fed AG, but there were no differences in peripheral blood lymphocyte numbers. Plasma levels of immunoglobulins were significantly greater for $\operatorname{IgG}(p<0.01)$ and $\operatorname{IgM}$ $(p<0.001)$ in pups from the dams fed AG than in the control pups. The supplementation of AG in the diets of lactating dams significantly elevated AG levels in the milk, and the increased AG in the milk subsequently stimulated certain immune responses in the pups. (Pediatr Res 36: 300-305, 1994)
Abbreviations
PBG, peripheral blood granulocyte
PBL, peripheral blood lymphocyte
TNF- $\alpha$, tumor necrosis factor- $\alpha$
LD, lactation day

Human colostrum and milk have been shown to possess a wide variety of immunologic factors (1). Immune factors in milk include cellular components such as lymphocytes, macrophages, and mononuclear cells and soluble components including immunoglobulins, lactoferrin, lysozyme, and other specific antibodies $(2,3)$ as well as essential nutrients. The list is not, however, inclusive of all potential immunoreactive components in milk. Other factors that might influence neonatal morbidity or immunocompetence have been found in vitro (4-6). Studies of infant mononuclear cells indicate that these factors have a role in vivo (7). Host defense against microbial infection in the newborn depends on the prompt and precise integration of cellular and humoral immune responses. The fetus has been in a sterile intrauterine environment, and at birth, the neonate has not yet developed a mature immune system (8). To deal with the hostile extrauterine environment, the newborn immune system must accomplish an adequate immunocompetence. Migliore-Samour

Received August 30, 1993; accepted March 25, 1994.

Correspondence and reprint requests: Suk Y. Oh, Ph.D., Department of Dictetics and Nutrition, University of Kansas Medical Center, Kansas City, KS 66160-7650.

Supported by the Clinical Nutrition Grant from the Speas Foundation, Kansas City, KS. and Jolles (9) have suggested that the evolution of immune function in the newborn may be influenced by milk components or their degradation products.

Alkylglycerols are glyceryl ether lipids that are structurally characterized by an ether linkage of a fatty acid attached to the $\alpha$-position of the glycerol backbone. Depending on the chain length and the number of double bonds, several derivatives of the lipid have been identified. The principal alkylglycerols include chimyl (hexadecyl), batyl (octadecyl), and selachyl (octadecenyl) alcohols (10). A small amount of alkylglycerol has been found in the living cells of most animal tissues, particularly in hematopoietic organs including bone marrow, spleen, liver, and lymphatic tissues and blood (11). A much greater content of ether lipids has been found in malignant tumors than in normal tissues (12), and the highest content of alkylglycerol was determined in the liver oil of Greenland sharks, the gray dog fish, and the rat fish (10). The glyceryl ether lipids were also found in human colostrum, human milk, and sheep's milk, and human milk contains nearly 10 times more alkylglycerol than cow's milk (13).

The biologic effects of alkylglycerol have been shown in both animals and human patients with cancers. Admin- 
istration of alkylglycerol to animals stimulated hematopoiesis including erythropoiesis, thrombocytosis, and granulocytosis (14-16). Treatment of uterine cervical cancer patients who were receiving radiation therapy with alkylglycerols significantly reduced the injuries accompanying the radiation toxicity and resulted in enhanced survival rate and survival time (17). A number of observations reported that alkylglycerols and alkyl lysophospholipids significantly activate cytotoxic macrophages, leading to enhanced Fc-receptor-mediated phagocytosis (18) and increase humoral immune response and delayed hypersensitivity reaction (19). Other studies showed that alkylglycerols inhibit the growth of primary tumors and the metastasis of the 3-Lewis lung carcinoma growing in $\mathrm{C}_{57} \mathrm{~B} 6$ mice (20). The present study was conducted to determine whether dietary alkylglycerols in lactating rats influence the ether lipid concentration in the milk of the lactating dams and to determine the effect of milk-borne alkylglycerols on the development of immune responses in suckling pups.

\section{METHODS}

\section{Animal and Care}

Sprague-Dawley female rats, 8-10 wk old, were purchased from Charles River Breeding Laboratories (Portage, MI) and bred by placing one female in a cage with one male until a vaginal wash was confirmed as sperm positive. This procedure was repeated until 48 females were found to be sperm positive. The day of spermpositive vaginal wash was designated as gestation $\mathrm{d} 0$ for each rat. Twelve pregnant females were randomly assigned to one of four dietary groups: a control and three treatment groups that differed in alkylglycerol levels. All animals were fed the control diet until parturition. The animals were housed in individual shoe box-style plastic cages that were designed to prevent access of the pups to the dam's feed, which contained different levels of alkylglycerols. The main characteristics of the cage are briefly described below. The dam's feed jars were placed in the front section of the breeding cage. A vertical Plexiglas divider with a hole large enough for the dam to pass through was placed in the middle of each cage, thus allowing the dam to reach the feed through the hole. The pups could not reach the feed because the hole was placed too high for them to climb through. However, the pups might have ingested glycerol ether lipids when they licked the dam's fur, which could have become contaminated by the alkylglycerols in the dam's diet. The animal cages were in a temperature-controlled room $\left(22^{\circ} \mathrm{C}\right)$ with a 12-h light-dark cycle, with light starting at $0600 \mathrm{~h}$. Rats were allowed feed and distilled water ad libitum. A fresh supply of diet was given daily. The pups were counted at birth, and litter sizes were adjusted to 12 or eight within $48 \mathrm{~h}$ of parturition. The animal experiment was conducted according to the guidelines for proper animal care established by the Institutional Animal Care and Use
Committee at the University of Kansas Medical Center, Kansas City, Kansas. the procedures used in the present study were approved by the Committee.

\section{Diets}

The basal diet was formulated according to the AIN-76 diet for rodents established by the American Institute of Nutrition Ad Hoc Committee on standards for nutritional status for animals. The AIN 76-diet meets the known nutritional requirements for growth of weanling rats and reproduction and lactation of adult rats (21). The basal diet was supplemented with alkylglycerols in the range of 10,50 , and $250 \mathrm{ppm}$. Synthetic alkylglycerols (chimyl, batyl, and selachyl glycerols) were purchased from Eastern Chemicals (Smithtown, NY) and solubilized in corn oil in the proportion of $30 \%$ chimyl, $28 \%$ batyl, and $42 \%$ selachyl glycerol. This mixture of three principal alkylglycerols closely resembles the alkylglycerol composition in human milk (15). The experimental diets were fed to the dams after parturition throughout the lactation period.

\section{Milk Expression}

During the lactation period, milk samples were expressed from each lactating dam on postpartum d 8, 16, and 24. For milk expression, lactating dams were separated from pups for $2 \mathrm{~h}$ before anesthesia with a rumpunketamine (1.25:3) mixture. Dams received an intraperitoneal injection of $0.1 \mathrm{~mL}$ of the anesthetic solution per 100 $\mathrm{g}$ of body weight. The milk production was stimulated by an intraperitoneal injection of $0.1 \mathrm{~mL}$ of oxytocin $(20$ $\mathrm{IU} / \mathrm{mL}$ ) per $100 \mathrm{~g}$ of body weight. The breast tissue was gently massaged with a warm, wet cloth. The nipples were then swabbed with a clean, warm cloth and milked with a suction apparatus consisting of a miniature glass funnel fitted to rubber tubing that was fitted into a 20gauge hypodermic needle inserted through the rubber stopper of a small collection bottle. A second needle was inserted into the stopper of the collection bottle and connected via rubber tubing to a vacuum source. After expression of milk, the dams were left in a warm cage for full recovery and then returned to their cages. Most of the dams nursed their pups normally, but two mutilated their pups after milk expression.

\section{Measurement of Litter Weight and Blood Collection}

On LD 8, 16, and 24, litter weight was determined and one third of the pups from each litter were anesthetized in a jar containing metofane to obtain blood samples by cardiac puncture; the blood was placed into vacutainers containing EDTA.

\section{Laboratory Procedures}

Complete blood counts. Blood samples taken from pups in the presence of EDTA were used for total and differential leukocyte counts by an automated STKR Coulter 
counter (Coulter Electronics, Inc., Hialeah, FL). When the instrument failed to count differential leukocytes for enumeration of PBG and PBL, then differential leukocytes were manually counted under a microscope (Olympus, Lake Success, NY) after staining the blood smear on a slide glass with Wright's stain.

Extraction and determination of milk alkylglycerol levels. The cream of pooled milk for each treatment group was prepared by centrifugation at $2000 \mathrm{rpm}$ for $20 \mathrm{~min}$. The milk fat was washed twice with saline to remove any residual sugars and proteins. Two $g$ of milk fat were used for extraction of alkylglycerols. A known amount of dodecylglycerol, an internal standard, was added to each extraction tube. The nonsaponifiable lipids were extracted by the procedures of Folch et al. (22). The fat samples were hydrolyzed in a mixture of $5 \mathrm{~mL}$ of $2 \mathrm{~N}$ methanolic $\mathrm{KOH}$ and $5 \mathrm{~mL}$ of chloroform for $2 \mathrm{~h}$ at $80^{\circ} \mathrm{C}$. Because the ether bond of alkylglycerols is nonsaponifiable, the ether lipids were directly extracted from the alkaline hydrolysate. The hydrolysate was first filtered through Whatman No. 1 filter paper, and an additional 5 $\mathrm{mL}$ of chloroform were added to each tube. To the filtrate, $5 \mathrm{~mL}$ of saline was added, vigorously mixed on a vortex, and briefly centrifuged to separate chloroform from aqueous methanol. The bottom layer of chloroform, containing the unsaponifiable lipids, including alkylglycerols, was carefully removed. The aqueous hydrolysate was extracted with an additional $10 \mathrm{~mL}$ of chloroform. The combined chloroform extracts $(20 \mathrm{~mL})$ were washed with saline to remove any contaminating soaps. The chloroform extracts were then dried over $\mathrm{MgSO}_{4}$, filtered, and evaporated under reduced pressure at a temperature not exceeding $30^{\circ} \mathrm{C}$.

Analysis of alkylglycerols. The dried nonsaponifiable lipids were dissolved in methylene chloride and applied to a column packed with activated alumina pretreated as previously described $(10,11)$. For the column chromatography, 0.25 to $0.5 \mathrm{~g}$ of nonsaponifiable lipids were applied to a column of $20 \mathrm{~g}$ of alumina, $10 \mathrm{~mm}$ in diameter and $150 \mathrm{~mm}$ in length. The methyl esters, carotenoids, squalene, and other substances of low polarity were eluted with methylene chloride. After 10 column volumes, cholesterol appeared, often together with orange substances. After some tailing of the cholesterol, the polarity of the solvent was increased by adding a $10 \%$ methanol solution to the methylene chloride. The glyceryl ether lipids were rapidly eluted without much tailing until 15 to 20 column volumes of the solvent mixture were passed through the column. The alkylglycerols were then converted into their trifluoroacetic derivatives. The qualitative and quantitative analyses of the glyceryl ether lipids were performed by gas chromatography (GC 14A, Shimadzu Scientific Instruments, Inc., Columbia, MD) using a packed column ( 4 feet $\times 1 / 4$-inch inner diameter) with a Dexsil 100 Supelcoport (Supelco, Bellafonte, PA). A GC 14A chromatogram of alkylglycerols isolated from a rat milk sample is shown in Figure 1.
Assays for immunoglobulins (IgG and IgM). Plasma concentrations of $\operatorname{IgG}$ and $\operatorname{IgM}$ were assayed using the ELISA with MAb (Kirkgaard \& Perry Labs, Gaithersburg, MD). The antigens (immunoglobulins) formed a complex with the MAb, which were fixed on the plate. Unbound antibodies were removed by aspiration and washing of the wells. Horseradish peroxidase was added to conjugate antibodies (i.e. anti-mouse IgG). The substrate $\left[2,2^{\prime}\right.$-azinobis(3-ethylbenz-thiazoline-6-sulfonic acid)] was then added to develop an orange-yellow color, which was proportional to the concentration of the complex formed. A complete Ig ELISA plate was suitable for nine assays with serial dilutions of each sample. A standard curve was constructed for each plate using pure immunoglobulins purchased from Sigma (St. Louis, $\mathrm{MO}$ ).

Assays for TNF- $\alpha$. TNF- $\alpha$ concentration in pups' plasma samples was determined using a commercial kit for ELISA (Genzyme Corp., Cambridge, MA).

Data analysis. Because the data were normally distributed, all results are presented as mean \pm SD. Data on milk levels of alkylglycerols, numbers of PBG and PBL in the pups' blood, and plasma levels of immunoglobulins were analyzed by an analysis of variance for repeated measures as previously described (23). The significance of difference was determined by using a $t$ test.

\section{RESULTS}

The ether lipid concentration in rat milk was approximately $0.03 \%$ of the milk fat. However, the rat milk was very high in milk fat content $(16.4 \%)$. Ether lipid concentration in swine bone marrow was $0.26 \%$ of bone marrow fat, which composed $12.1 \%$ of bone marrow. Concentrations of alkylglycerols in other samples were similar to the values reported previously (11).

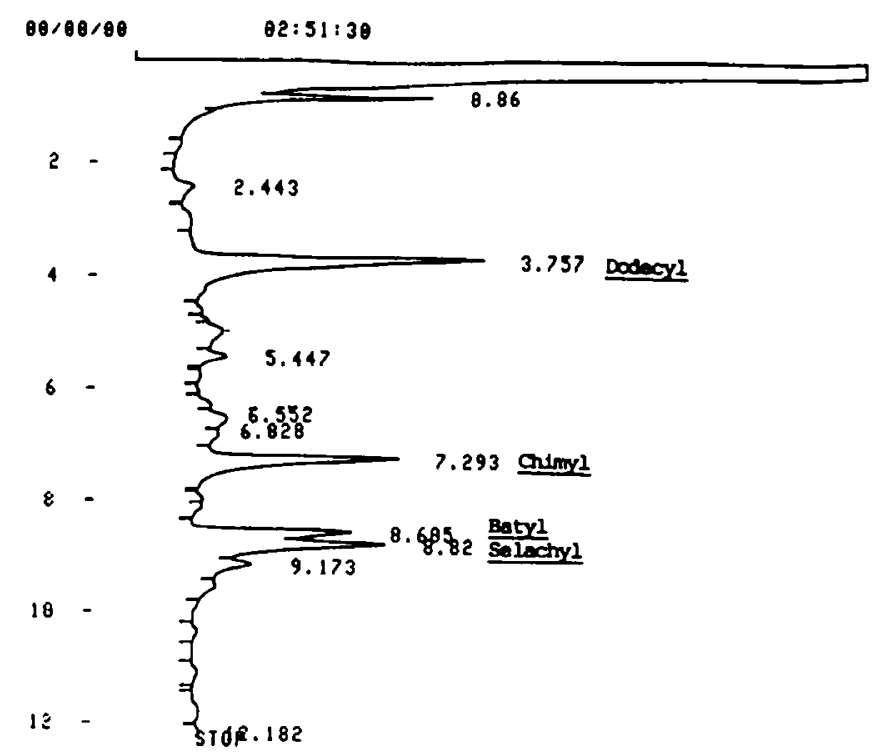

Figure 1. Gas-liquid chromatogram of the rat milk alkylglycerols. Stationary phase: Dexil 100 Supelcoport. Temperature program was in the range from 180 to $350^{\circ} \mathrm{C}$. A packed glass column 4 feet long with a $1 / 4$-inch inner diameter was used. 
Table 1 shows the changes in the pups' body weights during lactation on postpartum d 8,16, and 24. The pups' average growth rate was $2.37 \mathrm{~g} / \mathrm{d}$ for the control group and $2.43,2.32$, and $2.25 \mathrm{~g} / \mathrm{d}$ for the pups from dams fed diets containing 10,50, and $250 \mathrm{ppm}$ alkylglycerols, respectively. Although not statistically significant, the growth rates of the pups from the dams on the 250-ppm alkylglycerol diet appeared lower than that of the other groups.

The alkylglycerol concentrations in the milk samples were proportionally increased in response to dietary alkylglycerol levels for the dams, as shown in Table 2 . The alkylglycerol concentrations in the milk samples from control dams were 161.5, 173.9, and $181.4 \mu \mathrm{M} / \mathrm{L}$ $(54,58.2$, and $60.7 \mu \mathrm{g} / \mathrm{mL})$ of milk expressed on postpartum $\mathrm{d} 8,16$, and 24 , respectively, during the lactation period. The alkylglycerol concentrations in the milk from the dams fed 10,50, and $250 \mathrm{ppm}$ alkylglycerols increased approximately 40,100 , and $400 \%$, respectively, compared with that in the milk of the control dams.

There was no difference in the pups' PBL among the treatment groups receiving four levels of dictary alkylglycerols throughout the experiment. However, the numbers of pups' PBL are shown in Figure 2. The lymphocytes in general increased by approximately $50 \%$ during the lactation period as the pups grew. There were, however, no differences in lymphocyte numbers among the treatment groups. The numbers of granulocytes in pups' blood are shown in Figure 2. The granulocyte and lymphocyte numbers were gradually increased as the age of the pups increased. However, the granulocyte numbers in the pups from the mothers fed the diet containing 50 ppm were significantly greater $(p<0.05)$ compared with

Table 1. Effect of alkylglycerols in the dam's diets on pup's body weight*

\begin{tabular}{ccccc}
\hline $\begin{array}{c}\text { Dietary } \\
\text { alkylglycerol } \\
\text { levels (ppm) }\end{array}$ & $0(n=12)$ & $8(n=12)$ & $16(n=8)$ & $24(n=4)$ \\
\cline { 2 - 5 } & $6.3 \pm 0.6$ & $23.1 \pm 1.5$ & $41.9 \pm 2.4$ & $60.5 \pm 3.1$ \\
10 & $6.1 \pm 0.4$ & $22.9 \pm 1.3$ & $42.5 \pm 2.3$ & $62.0 \pm 3.2$ \\
50 & $6.0 \pm 0.5$ & $22.5 \pm 1.5$ & $40.7 \pm 2.8$ & $59.3 \pm 3.0$ \\
250 & $6.5 \pm 0.7$ & $22.0 \pm 2.1$ & $39.1 \pm 3.2$ & $58.3 \pm 4.4$ \\
\hline
\end{tabular}

* All values are means \pm SD (g) of four to 12 litters, depending on the number of days in lactation. $n$ indicates number of the litters that remained after the preceding euthanasia.

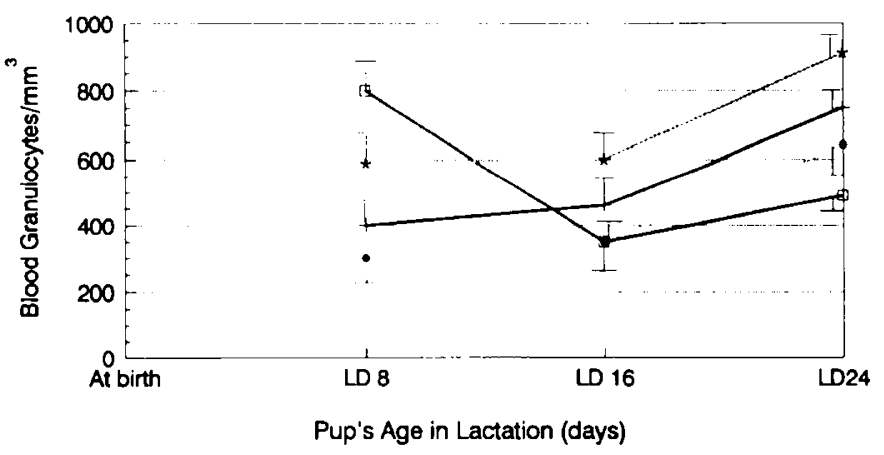

The concentrations of alkylglycerols in the diets were

- $0+10 \star 50 \div 250$ ppm respectively.

Figure 2. Effect of dietary alkylglycerols in lactating dams on development of PBG in pups. Results are expressed as means with SD for sextuplicate determinations.

the number of granulocytes in the control pups. The number of PBG of the pups from the dams fed $250 \mathrm{ppm}$ alkylglycerols was significantly greater $(p<0.01)$ on postpartum $d 8$ than that of the control pups but sharply declined during the rest of the lactation period.

The effect of alkylglycerols in the dams' diets on their pups' plasma immunoglobulin levels is shown in Table 3. Plasma IgG levels of the pups from the dams that ingested $10 \mathrm{ppm}$ alkyglycerols in the diet were significantly greater $(p<0.05)$ than those of the control pups. The plasma immunoglobulin levels of the pups from dams fed 50 and $250 \mathrm{ppm}$ alkylglycerols were also much greater $(p<0.01)$ than those of the control pups. When the lactating dams consumed the feed containing alkylglycerols, the plasma IgM levels of the pups were increased by a 2- to 4-fold magnitude, indicating that alkylglycerols activate the mature $\mathrm{B}$ lymphocytes and lead to the induction and proliferation of IgM. The plasma levels of TNF- $\alpha$ were determined by ELISA using a commercial kit (Genzyme Corp.), but measurable levels of TNF- $\alpha$ were not detected.

\section{DISCUSSION}

The presence of glyceryl ether lipids in human milk and cow's milk was first demonstrated by Hallgren and Larsson (11), who isolated glyceryl ethers from the milk cream. The investigators showed a surprisingly large

Table 2. Effects of dietary alkylglycerols in lactating rats on alkylglycerol levels in rat milk*

\begin{tabular}{|c|c|c|c|c|c|c|c|c|c|}
\hline \multirow{2}{*}{$\begin{array}{c}\text { Dietary } \\
\text { alkylglycerol } \\
\text { levels (ppm) }\end{array}$} & \multicolumn{3}{|c|}{ Chimyl-glycerol $(\mu \mathrm{m} / \mathrm{L})$} & \multicolumn{3}{|c|}{ Batyl-glycerol $(\mu \mathrm{m} / \mathrm{L})$} & \multicolumn{3}{|c|}{ Selachyl-glycerol $(\mu \mathrm{m} / \mathrm{L})$} \\
\hline & LD 8 & LD 16 & LD 24 & LD 8 & LD 16 & LD 24 & LD 8 & LD 16 & LD 24 \\
\hline 0 & $55.6 \pm 5.1$ & $56.9 \pm 3.5$ & $59.7 \pm 4.1$ & $53.1 \pm 4.4$ & $60.1 \pm 3.5$ & $62.4 \pm 3.5$ & $52.8 \pm 4.4$ & $56.9 \pm 5.0$ & $59.3 \pm 4.1$ \\
\hline $10 \dagger$ & $73.6 \pm 4.7$ & $79.3 \pm 7.3$ & $85.3 \pm 7.9$ & $76.3 \pm 6.4$ & $82.7 \pm 7.8$ & $87.3 \pm 9.3$ & $69.8 \pm 5.3$ & $73.8 \pm 6.7$ & $78.2 \pm 7.3$ \\
\hline $50 \ddagger$ & $99.2 \pm 3.8$ & $108.7 \pm 5.1$ & $113.1 \pm 7.3$ & $90.5 \pm 7.8$ & $105.0 \pm 8.9$ & $116.1 \pm 9.9$ & $102.1 \pm 8.8$ & $129.0 \pm 9.3$ & $136.0 \pm 11.1$ \\
\hline $250 \S$ & $219.0 \pm 8.8$ & $266.7 \pm 8.2$ & $275.2 \pm 9.5$ & $208.6 \pm 11.9$ & $253.3 \pm 13.6$ & $261.2 \pm 16.0$ & $204.3 \pm 12.8$ & $231.8 \pm 14.3$ & $238.8 \pm 17.8$ \\
\hline
\end{tabular}

* All values are means of triplicate determinations \pm SD. To convert $\mu \mathrm{m} / \mathrm{L}$ to metric units $(\mathrm{mg} / \mathrm{dL})$, multiply by 316.5 for chimyl, 344.6 for batyl, and 342.6 for selachyl alcohol.

$\dagger$ The mean values of alkylglycerol concentration in milk at this level of dietary alkylglycerol were significantly different from the control values $(p<0.05)$.

$\ddagger$ The mean values as described above on $50 \mathrm{ppm}$ dietary alkylglycerol were significantly different from the controls $(p<0.001)$.

$\S$ The mean values for $250 \mathrm{ppm}$ were significantly from the controls $(p<0.005)$. 
OH ET AL.

Table 3. Effects of dietary alkylglycerols in lactating dams on plasma immunoglobulin concentrations in pups*

\begin{tabular}{|c|c|c|c|c|c|c|}
\hline \multirow{2}{*}{$\begin{array}{c}\text { Dietary } \\
\text { alkylglycerol } \\
\text { levels (ppm) }\end{array}$} & \multicolumn{3}{|c|}{$\operatorname{IgG}(\mu \mathrm{m} / \mathrm{L})$} & \multicolumn{3}{|c|}{$\operatorname{IgM}(\mu \mathrm{m} / \mathrm{L})$} \\
\hline & LD 8 & LD 16 & LD 24 & LD 8 & LD 16 & LD 24 \\
\hline 0 & $8.0 \pm 0.53$ & $8.3 \pm 0.79$ & $12.6 \pm 0.53$ & $53.7 \pm 7.79$ & $69.5 \pm 4.84$ & $81.1 \pm 4.32$ \\
\hline 10 & $11.8 \pm 0.53 t$ & $13.8 \pm 0.47 \dagger$ & $14.6 \pm 0.61 \dagger$ & $182.1 \pm 14.74 \ddagger$ & $242.1 \pm 22.11 \ddagger$ & $310.5 \pm 11.58 \ddagger$ \\
\hline 50 & $16.8 \pm 0.55 \S$ & $21.3 \pm 0.56 \$$ & $23.3 \pm 0.48 \S$ & $476.8 \pm 36.84 \|$ & $542.1 \pm 24.21 \|$ & $655.8 \pm 42.11 \|$ \\
\hline 250 & $17.1 \pm 0.83 \$$ & $21.2 \pm 1.10 \S$ & $22.9 \pm 0.56 \S$ & $471.6 \pm 44.22 \|$ & $593.7 \pm 42.11 \|$ & $621.1 \pm 82.11 \|$ \\
\hline
\end{tabular}

* All values are expressed as means \pm SD of quadruplicate measurements. To convert $\mu \mathrm{m} / \mathrm{L}$ to metric units (mg/dL), multiply 15000 for IgG. For conversion of $\mathrm{nM} / \mathrm{L}$ to $\mathrm{mg} / \mathrm{dL}$, multiply by 94000 for IgM.

$\dagger$ The mean values of IgG in plasma of pups from the dams fed alkylglycerols were significantly different from the control values $(p<0.05)$.

$¥$ Significantly different from the controls $(p<0.005)$.

$\S$ Significantly different from the controls $(p<0.01)$.

$\|$ Significantly different from the controls $(p<0.001)$.

amount of glyceryl ethers, $0.19 \%$ and $0.1 \%$ of cream in human colostrum and milk, respectively, whereas cow's milk contains only $0.01 \%$ of the milk fat. We, for the first time, showed that rat milk contains alkylglycerols, which on average made up $0.03 \%$ of milk fat. The percentage value was lower than that in human milk $(0.1 \%)$ but greater than that in cow's milk $(0.01 \%)$. However, because the rat milk contained a greater percentage of fat $(16.4 \%)$ in comparison with human milk $(2.8 \%)$, the level of alkylglycerols in rat milk seems greater than that in human milk. The composition of glyceryl ether lipids in murine milk was $18.2,20.2$, and $19.3 \%$ on average for chimyl, batyl, and selachyl glycerol, respectively. The percentages of glyceryl ethers in human milk are 23.9 , 22.8 , and $33.8 \%$ for chimyl, batyl, and selachyl glycerol, respectively (11). The finding of glyceryl ethers in milk is interesting, because these compounds have also been found in fetal tissues and rapidly reproducing tissues such as bone marrow (13). Whether glyceryl ethers in milk play a role in the growth or the immunodevelopment of newborns is still open to question. Brohult (24) found that selachyl glycerol has a growth-stimulating effect on Lactobacillus lactis. Bodman and Maisin (25) isolated glyceryl ethers from perinephric fat of neonatal calves and meconium. The concentration fell rapidly after birth.

The supplementation of the lactating dams' diets with alkylglycerols significantly increased the ether lipid concentrations in the milk by approximately 36,94 , and $417 \%$ for the diets containing 10,50 , and $250 \mathrm{ppm}$ ether lipids, respectively. These data suggest that alkylglycerols in the diets were well absorbed by the intestinal mucosal cells. The relatively low responses of selachyl glycerol in the milk to the level in the dams' diets may be explained by the low purity (about $85 \%$ ) of the ether lipid. The increases in alkylglycerol content of the milk were dose dependent. The greater levels of ether lipids supplemented to the dams' diets resulted in greater concentrations of the lipids in the milk.

The alkylglycerols in the milk did not influence PBL in the pups' blood. The lymphocytes account for almost $90 \%$ of blood leukocytes, which gradually increased during the lactation period. The alkylglycerol supplementations to the diets for the lactating dams greatly $(p<0.05)$ raised the pups' PBG, as shown in Figure 2. The highest level $(250 \mathrm{ppm})$ of alkylglycerols markedly increased ( $p$ $<0.01$ ) the granulocytes on LD 8 but fell by LD 16 and remained low during the rest of the lactation period, suggesting that a long-term intake of $250 \mathrm{ppm}$ alkylglycerols may be toxic to the pups. The increase in the granulocytes may have been related to a shift between the marginal and circulating pools of granulocytes, rather than absolute increase in production.

The presence of additional glyceryl ethers in the milk significantly elevated plasma levels of $\operatorname{IgG}$ and $\operatorname{IgM}$ in the suckling pups. The mean plasma IgG level of the control pups was greatly increased $(p<0.05)$ from LD 8 to LD 24 , indicating that the pups synthesized more $\operatorname{IgG}$ as they grew. The plasma IgG levels of the pups were significantly elevated by the supplementations of alkylglycerols to the dams' diets containing $10 \mathrm{ppm}(p<0.05), 50 \mathrm{ppm}$ $(p<0.01)$, and $250 \mathrm{ppm}(p<0.01)$ ether lipids. There was no additional effect of alkylglycerols in the diets beyond $50 \mathrm{ppm}$. IgG composes approximately $70 \%$ of the total immunoglobulins in adult rat serum (26). Serum levels of IgG are, however, very low in newborn rats. Once they suckle, newborn rats' levels rise quickly because of the absorption of IgG from ingested milk via an IgG-Fc receptor on the gut epithelium (27). The increased plasma levels of $\operatorname{IgG}$ in the pups from the mothers fed alkylglycerols during lactation suggest that the increased alkylglycerols in milk could have stimulated production of IgG.

The plasma IgM levels in the pups from the dams on the control diet were very low during the lactation period. Ingestion of alkylglycerols by the lactating mothers raised the immunoglobulin concentrations by a multiple of $3(p<0.01)$ in those supplemented with $10 \mathrm{ppm}$ and by a multiple of $9(p<0.001)$ in those supplemented with 250 ppm. IgM represents about $10 \%$ of the total serum immunoglobulins and is largely confined to the intravascular pool (28) due to its larger size. IgM is the predominant antibody synthesized by the fetus. Although little immunoglobulin is produced in utero, exposure to immunogens may lead to an increased production of IgM antibodies by the fetus (26). The mechanism of the increased levels of plasma IgM in the pups from dams supplemented with alkylglycerols remains unknown. In anticipation of macrophage activation by alkylglycerols in the milk, plasma 
levels of TNF- $\alpha$ were determined in the pups' plasma samples. No TNF- $\alpha$ was detected in the pups' plasma samples acquired on LD 8, 16, and 24. The induction of TNF- $\alpha$ in response to antigens is very quick and lasts only a few hours unless an infection is present. The chronic ingestion of alkylglycerols via milk would not sustain TNF- $\alpha$ levels in blood even if the glyceryl ethers activate macrophages for a short period in early lactation.

In this study, we found that murine milk contains glyceryl ether lipids in amounts comparable to those in human milk. The supplementation of alkylglycerols in the diets of lactating dams greatly increased the concentration of ether lipids in milk in a dose-response manner. The increased levels of alkylglycerols in murine milk significantly increased the number of granulocytes in peripheral blood and plasma levels of immunglobulins, particularly IgM, in suckling pups. Results of the present study suggest that alkylglycerols in murine milk play a role in development of immune response in newborn rat pups.

Acknowledgments. The authors thank Peter L. Beyer for reading the manuscript. We gratefully acknowledge the assistance of Dr. Masahiro Chiga and the staff of the Hematology Clinical Laboratory of Kansas University Hospital for the total and differential leukocyte counts.

\section{REFERENCES}

1. Hanson LA, Winberg $J 1972$ Breastmilk and defense against infection in the newborn. Arch Dis Child 47:845-848

2. Orga SS, Weintraub D, Orga PL 1977 Immunologic aspects of human colostrum and milk. J Immunol 119:245-248

3. Prentice A 1987 Breast feeding increases concentrations of IgA in infants' urine. Arch Dis Child 62:792-795

4. Trapper D, Klagsbrun M, Neumann J 1979 The identification and clinical implications of human breast milk mitogen. J Pediatr Surg 14:803-808

5. Carpenter G 1980 Epidermal growth factor is a major growth-promoting agent in human milk. Science 210:198-199
6. Keller MA, Kid RM, Bryson YJ, Turner JL, Carter J 1981 Lymphokine production by human milk lymphocytes. Infect lmmunol 32:632-636

7. Stephens S, Brenner MK, Duffy SW, Lakhani PK, Kennedy CR, Farrant J 1986 The effect of breast-feeding on proliferation by infant lymphocytes in vitro. Pediatr Res 20:227-231

8. Quie PG 1990 Antimicrobial defenses in the neonate. Semin Perinatol 14:2-9

9. Migliore-Samour D, Jolles P 1988 Casein, a prohormone with an immunomodulating role for the newborn? Experientia 44:188-193

10. Hallgren B, Larsson S 1962 The glyceryl ethers in the liver oils of elasmobranch fish. J Lipid Res 3:31-38

11. Hallgren B, Larsson S 1962 The glyceryl ethers in man and cow. J Lipid Res $3: 39-43$

12. Snyder F, Wood R 1969 Alkyl and Alkyl-1-enyl ethers of glycerol in lipids from normal and neoplastic human tissues. Cancer Res 29:251-257

13. Hallgren B, Niclasson A, Ställberg G, Thorin H 1974 On the occurrence of $1-()$-alkylglycerols and 1-()-(2-methoxy alkyl) glycerols in human colostrum, human milk, cow's milk, sheep's milk, human red bone marrow, red cells, blood plasma and a uterine carcinoma. Acta Chem Scand B28:1029-1034

14. Edlund $T 1954$ Protective effect of d,1-a-octadecylglycerol ether in mice given total body $x$-irradiation. Nature 174:1102

15. Linman JW 1960 Hemopoietic effects of glyceryl ethers. III. Inactivity of selachyl alcohol. Proc Soc Exp Biol Med 104:703-706

16. Osmond DG, Roylance PJ, Webb AJ, Yoffey JM 1963 The action of batyl alcohol and selachyl alcohol on the bone marrow of the guinea pig. Acta Hematol 29:180-186

17. Brohult A, Brohult J, Brohult S, Joelsson I 1986 Reduced mortality in cancer patient after administration of alkoxyglycerols. Acta Obstet Gynecol Scand 65:779-785

18. Berdel WE 1987 Ether lipids and analogs in experimental cancer therapy. Lipids 22:970-973

19. Arnold B, Staber FG, Miller JFAP 1979 Lysolecithin analogs as adjuvants in delayed-type hypersensitivity in mice. II. Studies on the mode of action. Eur J Immunol 9:367-370

20. Berdel WE, Bausert WR, Weltzien HU, Modolell ML, Widmann KH, Munder PG 1980 The influence of alkyl-lysophospholipids and lysophospholipid-activated macrophages on the development of metastasis of 3-Lewis lung carcinoma. Eur J Cancer 16:1199-1204

21. American Institute of Nutrition 1977 Reports of the AIN Ad Hoc Committee on standards for nutritional status. J Nutr 107:1340-1348

22. Folch J, Lees M, Sloan-Slanley GH 1957 A simple method for the isolation and purification of total lipids from animal tissues. J Biol Chem 226:497-509

23. Peterson RG 1985 Design and Analysis of Experiments. Marcel Dekker, New York

24. Brohult A 1960) Alkoxyglycerols as growth-stimulating substances. Nature 188:591-592

25. Bodman J, Maisin JH 1958 The $\alpha$-glyceryl ethers. Clin Chim Acta 3:253-274

26. Hammarström L, Smith CIE 1985 Immunoglobulin isotype diversity its functional significance. In: French MAH (ed) Immunoglobulins in Health and Disease. MTP Press, Norwell, MA, pp 31-52

27. Burton DR 1985 Immunoglublin G: functional site. Mol Immunol 22:161-206

28. Burton DR, Gregory L 1986 Structure and function of immunoglobulins In French MAH (ed) Immunoglobulins in Health and Disease. MTP Press, Norwell, MA, pp 1-22 